\title{
A new approach for detecting sudden hypotension in hemodialysis by using dual-channel optical system
}

\author{
Younessi Heravi Mohamad Amin* \\ Department of Basic Sciences, North Khorasan University \\ of Medical Sciences, Bojnurd, Iran \\ a.younessi7@gmail.com \\ Golmakani Ebrahim \\ Department of Anesthesiology and Critical Care \\ Mashhad University of Medical Sciences, Mashhad, Iran \\ Joharinia Sima \\ Department of Electrical Engineering \\ Young Researchers and Elite Club, Bojnourd Branch \\ Islamic Azad University, Bojnourd, Iran
}

Received 4 August 2015

Accepted 17 February 2016

Published 7 April 2016

\begin{abstract}
Dialysis hypotension is one of the most prevalence symptoms of dialysis and occurs in $40 \%$ of treatment sessions. Detection and prediction of hypotension is important for the well-being of the patient and for optimizing treatment. The aim of this study was to construct optical system to monitor blood pressure (BP) continuously and without cuff in hemodialysis based on pulse transit time (PTT) method. To measure the BP changes, dual-channel optical system were developed. In this study, individuals were classified into two groups of normal and hemodialysis. In both groups, BP and consequently PTT were earned three times in different positions. After the initial calibration, the regression equation was drawn for each subject. In normal group, each subject was placed in the supine position and BP was measured both by designed system and sphygmomanometer cuff. During BP measurements, in addition to BP, blood pressure decline was also monitored by optical system. For hemodialysis group, the same measurement setup was adopted. In both groups, the error between cuff method and PTT was calculated. Correlation coefficients for $\mathrm{BP}_{\text {cuff }}$ vs $\mathrm{BP}_{\mathrm{PTT}}$ were calculated and Bland-Altman plot was performed for the normal and hemodialysis groups. In this study 16 subjects participated. The results for normal group showed that maximum difference between cuff method and the present method was $14 \mathrm{mmHg}$ and for dialysis group was $16 \mathrm{mmHg}$. Bland-Altman plot in normal group revealed limits of agreement from -13.98 to $13.18 \mathrm{mmHg}$. Considering hemodialysis group, limits of agreement were from
\end{abstract}

${ }^{*}$ Corresponding author.

This is an Open Access article published by World Scientific Publishing Company. It is distributed under the terms of the Creative Commons Attribution 4.0 (CC-BY) License. Further distribution of this work is permitted, provided the original work is properly cited. 
-15.94 to $13.88 \mathrm{mmHg}$. The correlation coefficient was 0.74 for normal group and was 0.72 for hemodialysis group. The proposed system can monitor BP continuously and diagnose sudden hypotension. So it can be recommended as a useful method to indicate hypotension and can be used for dialysis unit.

Keywords: Blood pressure monitoring; optical system; hypotension; hemodialysis.

\section{Introduction}

Body fluids contain water, electrolytes, proteins and other substances. The precise regulation of these fluids within a very narrow physiologic range is essential to life. Kidneys, as a part of their function, filter physiologically essential substances such as sodium and potassium. ${ }^{1}$ Normal kidneys function plays an important part in maintaining, homeostasis, the composition of internal body fluid. There are a variety of disorders affecting the kidneys function, resulting to renal failure. Renal failure is a condition in which the kidneys fail to remove metabolic end products from the blood. ${ }^{2}$ In most cases, patients with renal failure need hemodialysis. In hemodialysis water and dissolved solutes move from blood to dialysate, and allows the removal of several liters of excess fluid during a typical 2 to $4 \mathrm{~h}$ treatment. ${ }^{3}$ During the hemodialysis, patient may experience various side effects ranging from hypotension to visceral reaction such as nausea and vomiting. Hypotension is the most common side effect of hemodialysis and occurs in 30-40\% of treatment sessions. ${ }^{4}$ Detection and prediction of hypotension is important for the well-being of the patient and for optimizing treatment. Hypotensive episodes are a major complication of hemodialysis. Hypotensive episodes can be responsible of acute vascular complications such as myocardial ischemia, ischemic cerebro-vascular accidents, venous thrombosis (retina vein), intestinal ischemia and the aggravation of lower member arthritis. ${ }^{5}$ The main causes of dialysis induced hypotension are age, hypovolemic (rapid ultrafiltration) and coexisting decrease autonomic neuropathy, cardiovascular diseases and diabetes. ${ }^{6}$

With this respect monitoring the blood pressure (BP) during hemodialysis would be a challenge for medical staff. Using traditional methods of controlling BP such as manual sphygmomanometer cuff is practically associated with several problems. For instance, it fails to provide continues BP monitoring. Accordingly many studies have recently attempted to design noninvasive continuous $\mathrm{BP}$ monitoring device. ${ }^{7-9}$ Although Tonometric, Vascular Unloading and Finapres methods have been developed and even in some cases released to market, a substantial problem, using cuff, has been remained as obstacle needs to be resolved. ${ }^{10,11}$ Therefore, designing a BP controlling device without cuff has drawn the attention of many researchers during the recent years. Impedance plethysmograph (IPG) with pulse sensor is a method based on blood volume that due to its complexity has been less used in clinics or hemodialysis unit. ${ }^{12}$ Another method is near infrared charge-coupled device (NIR CCD) camera. This method evaluates the relationship between the changes of vessel diameter and the BP. It is based on the changes of vessel diameter detected by NIR CCD camera in each cardiac cycle. NIR CCD is still in infancy and its adequacy has not been documented. ${ }^{13}$ Pulse transition time (PTT) is another method, acting based on continuous recording of ECG and pulse wave. The main features of PTT method including safety, noninvasive and continuous BP monitoring has been documented in a vast array of studies, ${ }^{14-18}$ but ECG recording impose another set of the complexity to hemodialysis. This study aimed to develop a system for continuous monitoring of $\mathrm{BP}$ in patients undergoing hemodialysis. This system is based on the PTT method, but uses two pulse sensors to take PTT without requiring the ECG signal.

\section{Material and Methods}

\subsection{Estimating blood pressure}

The Bramwell-Hill equation describes how pulse wave velocity (PWV) relates to arterial dispensability. ${ }^{19}$

$$
\mathrm{PWV}=\sqrt{\frac{V \Delta P}{\rho \Delta V}},
$$


where $\Delta p$ and $\Delta V$ are the changes of blood pressure and volume, $\rho$ is the blood density. PWV relation by PTT was shown in Eq. (2). ${ }^{8}$

$$
\mathrm{PWV}=L / \mathrm{PTT} \text {. }
$$

$L$ is the distance the pulse travels (roughly equals to the arm length). It is well known that the relationship between vascular volume $(V)$ and transmural pressure $(P)$, is commonly fitted as a sigmoid curve. $^{20}$

$$
V=\frac{a}{1+e^{b p}}
$$

By substituting Eq. (1) to Eq. (2) and re-arranging the equation with Taylor expansions, we obtain the relationship between PWV or PTT and blood pressure $(P)$.

$$
\mathrm{PWV}=\sqrt{\frac{e^{b P}+1}{\rho b}}=\frac{1}{\sqrt{\rho b}} \frac{\sqrt{2}}{\left(1-\frac{b P}{4}\right)} \equiv \frac{1}{c P-c / 4},
$$

$$
\mathrm{PTT}=L(c P-c / 4) \rightarrow P=A+B(\mathrm{PTT}) .
$$

This derived equation suggests that $\mathrm{BP}$ is linearly related to PTT and the coefficients $A$ and $B$ could be achieved by curve fitting based on the PTT and $\mathrm{BP}$ at different physical situations. ${ }^{17}$ In this paper, PTT was defined as the time between the two peaks of pulse signals.

\subsection{Experiments}

In this study, individuals were classified into two groups of normal and hemodialysis. In both groups, there were four men and four women. For continuous measurement of BP, the coefficients (Eq. (4); $A$ and $B$ ) are needed in addition to the continuous extraction of PTT. BP was measured three times in $0^{\circ}$ and $\pm 90^{\circ}$ of the right hand (Fig. 1) and PTTs were earned in these conditions. For the calibration, the linear relation between the measured PTTs and BPs was obtained for each subject based on three trials of measurements. To prevent cuff inflation problems, a time interval of $5 \mathrm{~min}$ was considered between each measurement. Linear equation was obtained based on three repeated measurements in different angles for each subject. Linear regression was performed using the standard linear regression model to fit a linear function for the set of data pairs. Based on this function, the relation between

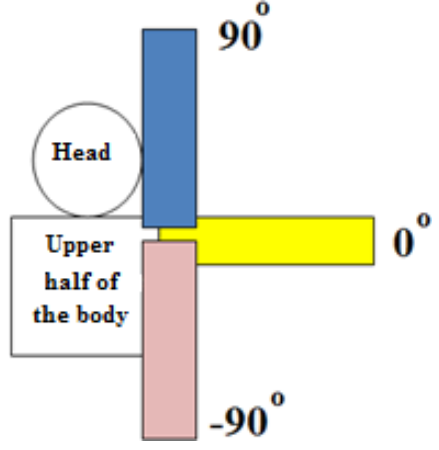

Fig. 1. The calibration process for detecting linear function by using three trials of measurements.

$\mathrm{BP}$ and PTT could be expressed as follows:

$$
\text { Psys }=A+B(\mathrm{PTT}) \text {. }
$$

So $A$ and $B$ coefficients were obtained based on the three-time measurement of PTT and BP. Linear regression was calculated and coefficients were determined separately for each set of data obtained from each individual. Figure 2 shows a graph of function PTT-BP for one subject.

In normal group, after calibration process, each subject was immediately placed in the supine position for $40 \mathrm{~min}$ and blood pressure was measured both by designed system and sphygmomanometer cuff. When subject was in the supine position, blood pressure measurements were repeated with a time interval of $5 \mathrm{~min}$ using designed system and cuff. For each measurement, the blood pressure $\left(P_{\mathrm{PTT}}\right)$ was calculated using Eq. (4). This process was immediately followed by cuff inflation to measure cuffbased blood pressure $\left(P_{\text {cuff }}\right) . P_{\text {PTT }}$ was compared to $P_{\text {cuff }}$ and the error was computed for each eight measurements (that were obtained during a $40 \mathrm{~min}$ observation with $5 \mathrm{~min}$ intervals). Absolute error and relative error were calculated as follows:

pressure_difference $(i)$

$$
\begin{aligned}
& =\left|P_{\mathrm{CUFF}}(i)-P_{\mathrm{PTT}}(i)\right|, \quad \text { relative_error }(i) \\
& =\frac{\left|P_{\mathrm{CUFF}}(i)-P_{\mathrm{PTT}}(i)\right|}{P_{\mathrm{CUFF}}(i)} \times 100, \quad i=1,2, \ldots, 8 .
\end{aligned}
$$

During BP measurements, in addition to systolic blood pressure, blood pressure decline was also monitored by optical system. To compute blood pressure decline, individual's BP was measured in static condition. BP measurement in static condition is defined as the first measurement in supine position and considered as the reference BP. The reference PTT level was also computed based on 

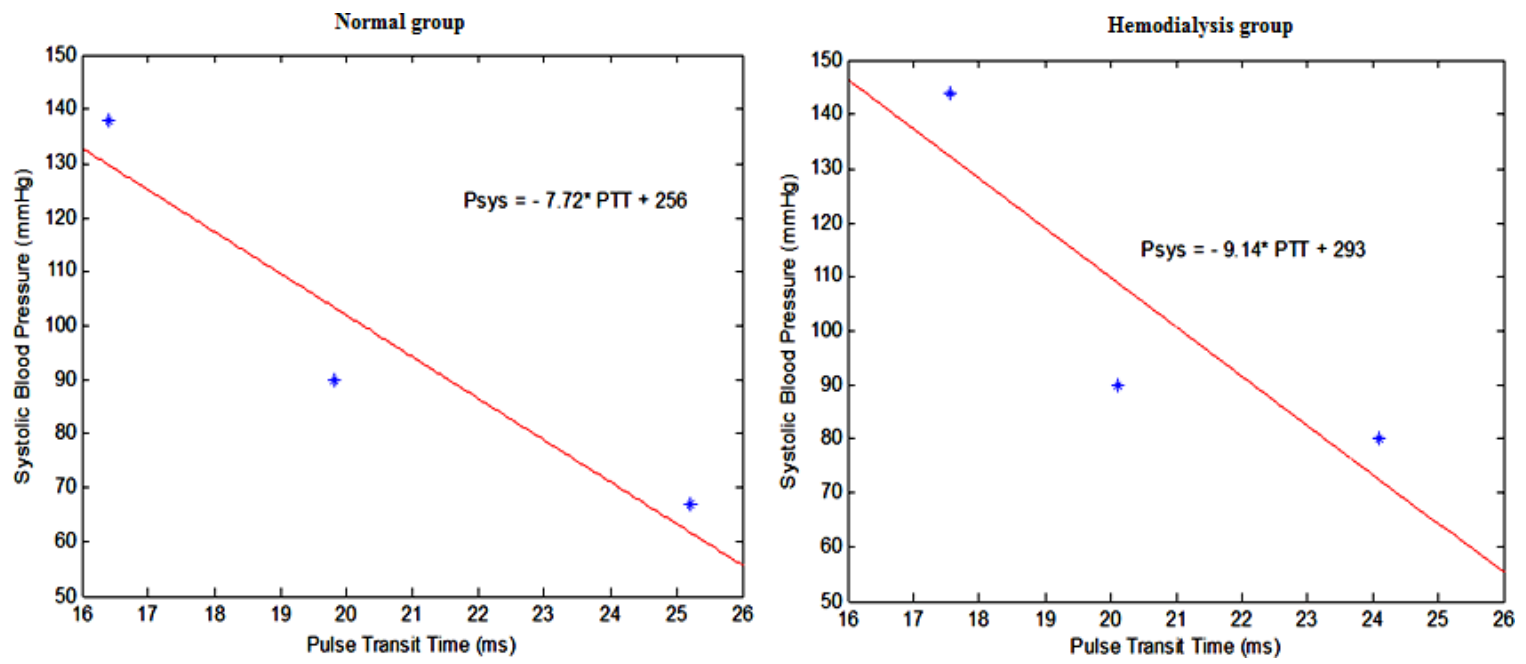

Fig. 2. Linear function for the set of data pairs in calibration process.

individual's coefficients $(A$ and $B$ ) and the reference BP. When PTT was applied to the Eq. (5), corresponding BP value could be calculated. During the experiment, PTTs-based BPs were compared with the reference value and the pressure difference was displayed. During the experiment, PTTs were acquired from $10 \mathrm{~s}$ after the cuff-deflation.

For hemodialysis group, the same measurement setup was adopted. Data was acquired during morning dialysis sessions for $40 \mathrm{~min}$ (20 min at the beginning as well as $20 \mathrm{~min}$ at the end of treatment). No actions were taken to increase or decrease BP and no instructions were given to the patient. The reference BP and PTT were measured before carrying out the dialysis process. During the process, PTTs and also BPs were compared with the reference values and the pressure decline was displayed. All cuff-based $\mathrm{BP}$ measurements were done by digital brachial BP device (Citizen, BK202) on the right upper arm.

Correlation coefficients for $\mathrm{BP}_{\text {cuff }}$ vs $\mathrm{BP}_{\mathrm{PTT}}$ were calculated for the normal and hemodialysis groups. In addition, a Bland-Altman plot was done. In the Bland-Altman plot, the mean value calculated from corresponding $\mathrm{BP}$ values that were obtained from both methods, is plotted against the corresponding difference between the mean $\mathrm{BP}_{\text {cuff }}$ and the $\mathrm{BP}_{\mathrm{PTT}}$ $\left(\mathrm{BP}_{\text {cuff }}-\mathrm{BP}_{\mathrm{PTT}}\right)$. The agreement limits were defined by mean \pm 1.96 of the standard deviation (SD) of the differences. The plot helps to assess the disagreement between the two methods of BP measurement. ${ }^{21}$ All statistical analyses were performed using Statistics toolbox in Matlab software version 2012a. A significance level of $p<0.05$ was considered to be significant.

\subsection{Optical system for blood pressure monitoring}

In designing this system, two infrared optical sensors (two optical transmitters) with $915 \mathrm{~nm}$ wavelengths and two optical receivers were employed. The optical transmitters were located at a distance of $5 \mathrm{~mm}$ from the optical receiver. The distance between two transducers was defined $55 \mathrm{~mm}$. This system has been patented in the Iranian Patent Organization under the Declaration No 81088. The analog filter and gain circuit were considered to have an appropriate signal. The output of the designed analog circuit was inserted into an analogto-digital converter of ATMEGA 32 microcontroller. For preprocessing on the signal, the output of the optical receiver was inserted in an instrumentation amplifier (AD620). Since pulse signal has DC frequency components to $30 \mathrm{~Hz}$ frequency, to eliminate low frequency distortions caused by skin absorption, bone and other nonpulsatory tissues a passive high pass filter with $0.5 \mathrm{~Hz}$ cut off frequency, and to eliminate high frequency noises greater than $30 \mathrm{~Hz}$ a passive low pass filter as well as two active low pass filters of second degree with $20 \mathrm{~Hz}$ cut off frequency were employed. Moreover, to have a signal with an appropriate range, an amplifier was utilized with variable gain. While recording, the recorded signal might have positive voltage level as well as negative voltage level and since a microcontroller is not possible with negative voltage level, in the last level of the circuit a level shifter is used to shift voltage level into an appropriate range $0-5 \mathrm{~V}$ positive voltage level. ${ }^{17}$ Figure 3 shows the block 


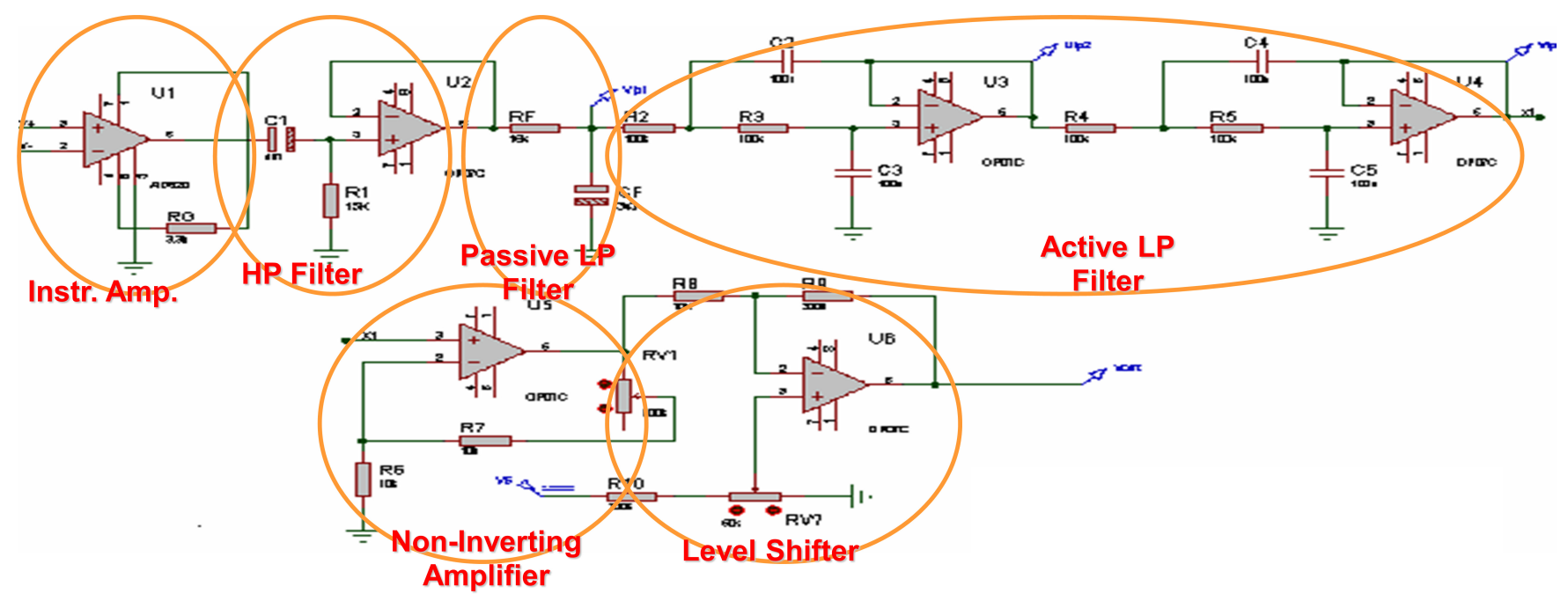

Fig. 3. The block diagram of the main participants of the designed amplifier in one channel.

diagram of the main participants of the designed amplifier in one channel. By detecting the peak of signals by controller, PTT was calculated continuously. Sampling frequency was considered as $50 \mathrm{~Hz}$. Figure 4 shows BP measurement by the designed system in hemodialysis unit. The two optical transducers which have interspaced $55 \mathrm{~mm}$ fixed on right middle finger (finger capillary network). To prevent motion artifact, optical amplifier and analog circuits were located on the wrist.

\subsection{Subjects}

Sixteen subjects participated ranging from 22 to 56 and eight were males and eight were females. For all

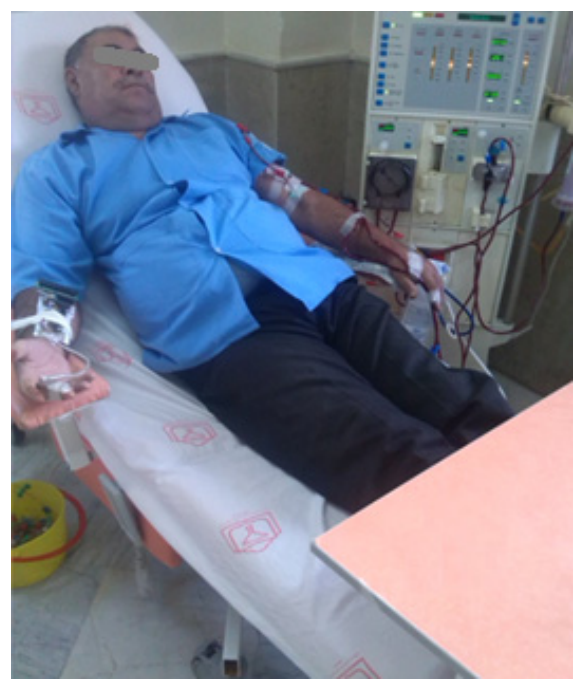

Fig. 4. Blood pressure measurement by the present method for one hemodialysis patient. of the subjects, the mean systolic blood pressure was $119.82 \pm 11.46 \mathrm{mmHg}$, the mean heart rate $81.75 \pm$ $8.48 \mathrm{BPM}$, and the average height $1.64 \pm 0.08 \mathrm{~m}$. The data was collected in skill lab of medicine school and dialysis unit in Imam Ali Hospital of North Khorasan University of medical sciences, in normal subjects and hemodialysis group, respectively. All subjects were sufficiently informed how to register data and steps in compliance with the basic principles register. This study has been approved in ethics committee of North Khorasan University of Medical Sciences in 2013/Jul/13 and complies with the Declaration of Helsinki and informs consent has been obtained from the subjects.

\section{Results}

Table 1 shows the relation between PTT and systolic pressure with correlation coefficient and error calculation in normal group. The results of Table 1 showed that absolute error between cuff method and PTT was $8.38 \pm 4.23 \mathrm{mmHg}$ and the error percentage was calculated as $7.51 \pm 3.48 \%$. Also the individual $(n=8)$ correlation coefficients of $\mathrm{BP}_{\mathrm{PTT}}$ vs $\mathrm{BP}_{\text {cuff }}$ varied between $R=0.61$ and $R=0.84$. Figure 5 shows the plot of $\mathrm{BP}_{\text {cuff }}$ and $\mathrm{BP}_{\mathrm{PTT}}$ and the regression for this group. Figure 5 shows the correlation coefficient for data of all eight subjects and measurements. The correlation coefficient was $R=0.7479$ and it was significant $(p<0.05)$. Figure 6 shows the BlandAltman plot of the normal group. Bland-Altman plots on the basis of the linear function revealed limits of agreement of -13.98 to $13.18 \mathrm{~mm} \mathrm{Hg}$. 
Table 1. The correlation coefficient between SBP and PTT with error calculation in normal group (f: female and $\mathrm{m}$ : male).

\begin{tabular}{|c|c|c|c|c|}
\hline Subject & $\begin{array}{c}\text { Correlation } \\
\text { coefficient }(R)\end{array}$ & $\begin{array}{l}\text { Difference } \\
\text { of pressure } \\
\text { between } \\
\text { two methods }\end{array}$ & Error (\%) & $\begin{array}{c}\text { Pressure } \\
\text { decline }\end{array}$ \\
\hline $1(\mathrm{f})$ & 0.72 & $8.53 \pm 3.62$ & $6.86 \pm 3.05$ & $5.3 \pm 3.22$ \\
\hline $2(\mathrm{f})$ & 0.81 & $9.21 \pm 4.06$ & $7.05 \pm 3.49$ & $5.26 \pm 3.66$ \\
\hline $3(\mathrm{f})$ & 0.68 & $7.96 \pm 4.81$ & $6.78 \pm 3.88$ & $6.24 \pm 4.11$ \\
\hline $4(\mathrm{f})$ & 0.84 & $9.54 \pm 3.84$ & $7.26 \pm 3.04$ & $7.21 \pm 3.86$ \\
\hline $5(\mathrm{~m})$ & 0.69 & $7.53 \pm 3.25$ & $6.15 \pm 2.86$ & $5.66 \pm 4.36$ \\
\hline $6(\mathrm{~m})$ & 0.61 & $10.11 \pm 4.02$ & $8.94 \pm 3.11$ & $7.15 \pm 3.45$ \\
\hline $7(\mathrm{~m})$ & 0.78 & $9.15 \pm 3.68$ & $8.21 \pm 2.86$ & $6.89 \pm 4.11$ \\
\hline $8(\mathrm{~m})$ & 0.82 & $7.81 \pm 4.03$ & $6.34 \pm 4.01$ & $7.69 \pm 4.15$ \\
\hline
\end{tabular}

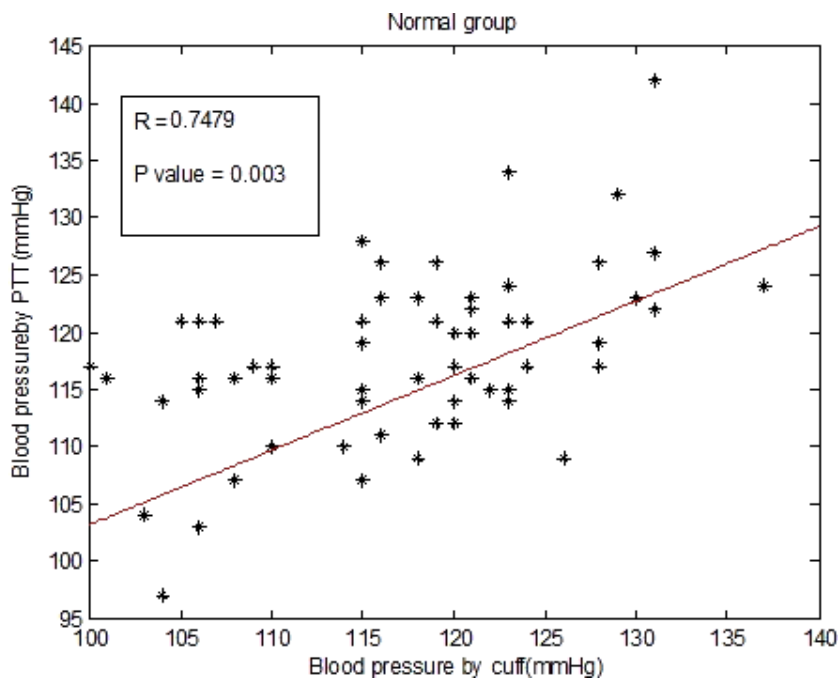

Fig. 5. Plot of systolic BP measured by cuff vs systolic blood pressure calculated from the pulse transit time of the normal group. The straight line represents linear regression (see inserted $R$ and $P$ value).

Table 2 shows the results of hemodialysis group. The results of Table 2 showed that absolute error and error percentage between cuff method and PTT were $8.61 \pm 4.17 \mathrm{mmHg}$ and $9.08 \pm 3.58 \%$, respectively. In this group, correlation coefficient varied between 0.58 and 0.80 . Figure 7 shows the plot of $\mathrm{BP}_{\text {cuff }}$ and $\mathrm{BP}_{\mathrm{PTT}}$ and the regression for this group. The correlation coefficient for data of all eight subjects and measurements was $R=0.7283$ and it was significant $(p<0.05)$. Figure 8 shows the Bland-Altman plot of the hemodialysis group. Bland-Altman plots on the basis of the linear function revealed limits of agreement of -15.94 to $13.88 \mathrm{mmHg}$. The more scatter was observed at the higher end of $\mathrm{BP}$ values.

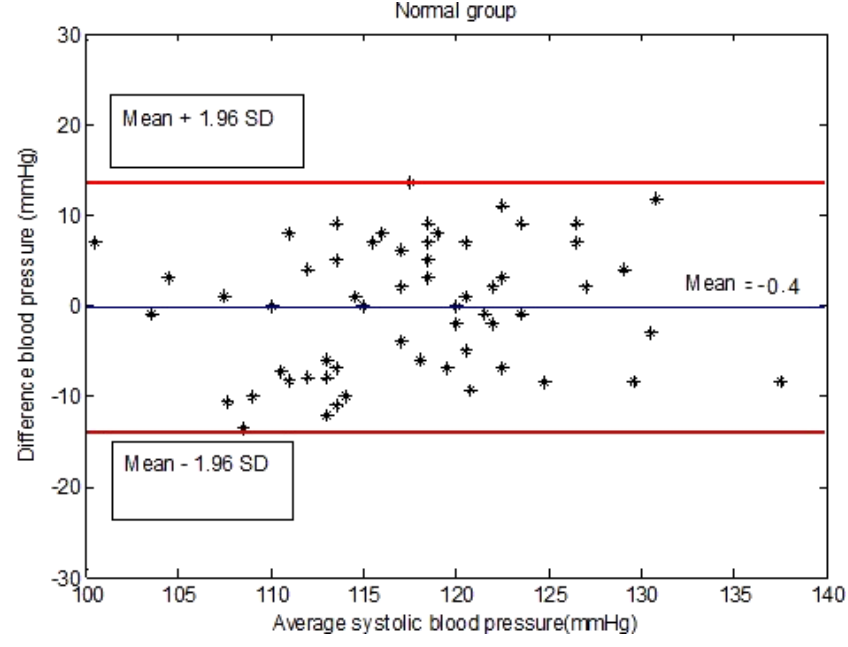

Fig. 6. Bland-Altman plots demonstrating the overall agreement between the $\mathrm{BP}_{\text {cuff }}$ and the $\mathrm{BP}_{\text {РTT }}$ in all eight sets of data by using each normal subject's individual linear function parameters. The outer lines indicate the limits of agreement, the inner line represents the mean difference.

In this study, the error percentage in hemodialysis was more than normal group. The lowest value of correlation coefficients was calculated 0.61 for normal and 0.58 for hemodialysis group. For normal group, the maximum difference and highest error percentage were $14.38 \mathrm{mmHg}$ and $9.74 \%$, respectively. Maximum difference and error for hemodialysis were obtained $16.21 \mathrm{mmHg}$ and $11.85 \%$, respectively. There was no significant relation in error and correlation coefficient between male and female in both groups. Maximum pressure decline in normal subjects was $10 \mathrm{mmHg}$ and for hemodialysis was $34 \mathrm{mmHg}$. In hemodialysis group, hypotension

Table 2. The correlation coefficient between SBP and PTT with error calculation in hemodialysis group. In * subjects, difference of pressure and error percentage between cuff method and PTT were calculated just before occurrence of hypotension (f: female and m: male).

\begin{tabular}{|c|c|c|c|c|}
\hline Subject & $\begin{array}{c}\text { Correlation } \\
\text { coefficient }(R)\end{array}$ & $\begin{array}{c}\text { Difference } \\
\text { of pressure } \\
\text { between two } \\
\text { methods }\end{array}$ & Error (\%) & $\begin{array}{c}\text { Pressure } \\
\text { decline }\end{array}$ \\
\hline $1(\mathrm{f})$ & 0.76 & $7.24 \pm 4.22$ & $6.02 \pm 3.24$ & $8.44 \pm 4.11$ \\
\hline $2(\mathrm{f})$ & 0.71 & $8.45 \pm 3.71$ & $6.97 \pm 3.06$ & $5.93 \pm 3.76$ \\
\hline $3(\mathrm{f})$ & 0.58 & $10.38 \pm 3.36$ & $8.69 \pm 3.55$ & $6.43 \pm 2.11$ \\
\hline $4(\mathrm{f})$ & 0.68 & $8.78 \pm 4.15$ & $7.02 \pm 3.34$ & $6.17 \pm 3.21$ \\
\hline $5(\mathrm{~m})^{*}$ & 0.72 & $9.38 \pm 3.71$ & $7.56 \pm 3.55$ & $>30$ \\
\hline $6(\mathrm{~m})$ & 0.66 & $9.06 \pm 4.21$ & $6.57 \pm 6.63$ & $7.33 \pm 3.51$ \\
\hline $7(\mathrm{~m})$ & 0.80 & $8.88 \pm 3.61$ & $6.99 \pm 2.79$ & $8.23 \pm 3.78$ \\
\hline $8(\mathrm{~m})^{*}$ & 0.75 & $8.29 \pm 4.26$ & $7.24 \pm 3.43$ & $>30$ \\
\hline
\end{tabular}




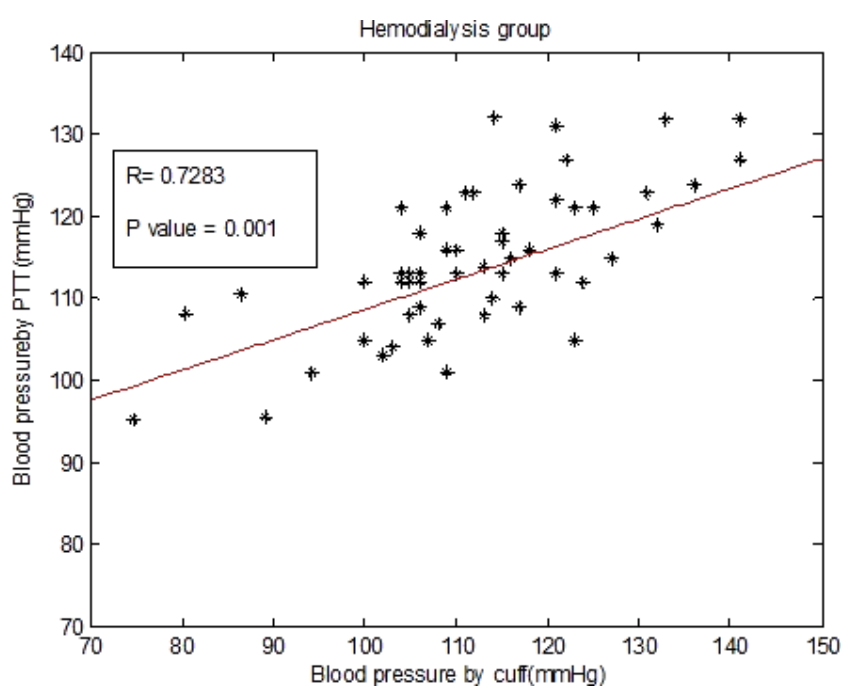

Fig. 7. Plot of systolic BP measured by cuff versus systolic blood pressure calculated from the pulse transit time of the hemodialysis group. The straight line represents linear regression (see inserted $R$ and $P$ value).

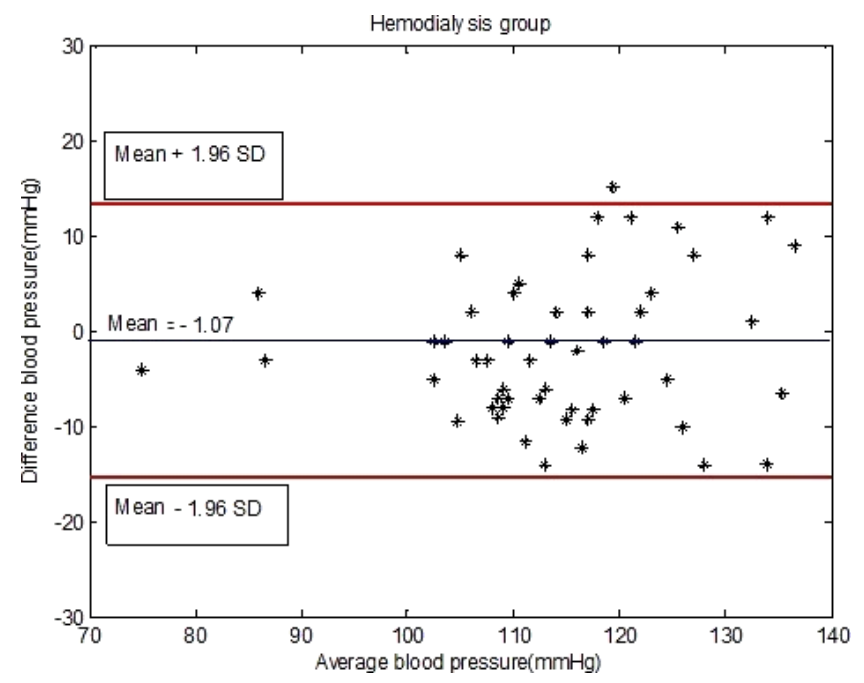

Fig. 8. Bland-Altman plots demonstrating the overall agreement between the $\mathrm{BP}_{\text {cuff }}$ and the calculated $\mathrm{BP}$ as a linear function of PTT in all eight sets of data by using each patient's individual linear function parameters.

occurred in two subjects and systolic BP decreased more than $30 \mathrm{mmHg}$. Therefore, hemodialysis operation and data collection were stopped. The pressure decline was detected and shown by the designed system in the both subjects.

\section{Discussion}

This study presents a novel system to measure continuous $\mathrm{BP}$ in hemodialysis patients. The results for normal group showed that maximum difference between cuff method and the present method (max $\left.\left[\mathrm{P}_{\text {cuff }}-\mathrm{P}_{\mathrm{PTT}}\right]\right)$ was $-14.38 \mathrm{mmHg}$ and for dialysis group was $+16.21 \mathrm{mmHg}$. The correlation coefficient was 0.747 for normal group and was 0.728 for hemodialysis group. For normal group, BP was measured with less error and higher accuracy as compared to hemodialysis group. The overall ability to predict BP values from PTT was analyzed using Bland-Altman plots of the calculated BP values obtained from the both groups. Bland-Altman plot in normal group revealed limits of agreement from -13.98 to $13.18 \mathrm{mmHg}$. Considering hemodialysis group, limits of agreement were from -15.94 to $13.88 \mathrm{mmHg}$. In this analysis, we observed slightly better limits of agreements for the normal as compared to the hemodialysis group.

In hypotension, a typical reduction in SBP is $30 \mathrm{mmHg}$ or more. ${ }^{4}$ In the current study, for two patients, hypotension occurred which was diagnosed by our system in the both cases. The overall incidence of hypotension during dialysis is $30-40 \%$ while we observed only two events in eight persons (25\%) which was unexpectedly lower than other studies. Ahlstrom et al. evaluated the pulse wave transit time (PWTT) technique for continuous monitoring of $\mathrm{BP}$ during hemodialysis treatment. But in this study, PTT was measured based on pulse wave and ECG signals and the method was more complicated than the present one. The results of Ahlstrom's study showed that pressure was estimated with a higher correlation with PTT. ${ }^{26}$ Our results were in good agreement with the aforementioned report while we did not use ECG for continuous BP. In studies that calculate PTT based on QRS complex extraction from the ECG as a time reference, this may not be the optimum reference to use as stated by the previous studies. The QRS complex is an electrical signal, a depolarization event which represents the point of expulsion of blood from the left ventricle. It is believed that there may be disparities regarding the iso-volumetric contraction period of the left ventricle, making it an unsuitable time reference. ${ }^{27}$ So, this study did not use ECG as the time reference.

Continuous measurement of $\mathrm{BP}$ was done in several studies based on PTT and without ECG in ways similar to our study. Kim et al. developed a wrist type device for the continuous noninvasive BP monitoring based on PTT. The device could connect to the PDA phone by wireless connection. 
Its error rate was higher than the commercial products, but it was a novel attempt which could approach the reliability of traditional $\mathrm{BP}$ measurement devices. ${ }^{28}$ Zakaria et al. recorded the values of PWTT and SBP in three experiments. According to the results of the experiments, three different mathematical equations for calculation of SBP from PWTT were obtained. The accuracy of derived $\mathrm{BP}$ values was low in comparison with the conventional method. ${ }^{29}$ In this study, BP was estimated based on PTT by the linear function but a number of authors obtained a nonlinear relationship between PTT and BP, ${ }^{16-18}$ while others reported linear relations which was in consistent with our study. ${ }^{20,22}$ In the present study, SBP was used to monitor BP based on PTT, because several studies have shown that SBP could be measured more accurately than diastolic blood pressure. ${ }^{16,20,22}$ The correlation between SBP and PTT was found to be $R=0.74 \pm 0.08$ for the normal and $0.7 \pm 0.07$ for the hemodialysis subjects. A brief summary of the results in previous studies ${ }^{22-25}$ gives a mean correlation coefficient of 0.63 which is in the range $0.33-$ 0.81 . This variability emphasizes the well-known difficulty in estimation of SBP by transit time methods without preceding calibration.

In this study, two pulse-sensors were used along an artery to measure the PTT. Our optical system was light $(80 \mathrm{gr})$ and it was applied on patient hand for a short period of time (two periods of $20 \mathrm{~min}$ ). There were no complaints from the participated patients regarding the device weight. The subjects were requested to fix the right hand as much as possible because the motion artifact and hand position result in $\mathrm{BP}$ variations. In our cases, unusual changes in BP and PTT caused by the movement were not considered. So, in future studies, hand position has to be monitored carefully at all time with the information of hand position and the system can correct the value of BP automatically. Prediction accuracy depends on the accuracy of the initial calibration. Initial calibration was done for each subject as physiological parameters such as the blood density and the stiffness of the arterial wall vary among the subjects. In the current study, we defined PTT as the time interval of the two peaks of pulse signals because it was much easier to detect for us in comparison to other part of signal. In the future studies, other signal parts should be used and the results should be compared to the results of the current study. Due to the small number of subjects, these results are preliminary and have to be confirmed by studies with more subjects.

\section{Conclusions}

The aim of this study was to detect sudden hypotension in hemodialysis patients by optical blood pressure monitoring system. The system was based on the PTT method but different from other systems which use ECG and pulse signals to obtain the PTT. The results showed that maximum error between the cuff method and the present method was $16 \mathrm{mmHg}$. So, the proposed system can monitor blood pressure continuously and diagnose sudden hypotension. Monitoring blood pressure with the designed system can be recommended as a useful method to indicate hypotension and can be used for dialysis unit.

\section{References}

1. J. T. Augirdas, "Pathophysiology of dialysis hypotension: An update," Am. J. kidney Dis. 38(4), S11-S17 (2001).

2. C. Ronco, "The haemodialysis system: Basic mechanisms of water and solute transport in extracorporeal renal replacement therapies," Nephrol. Dial. Transplant. 13(90006) 3-9 (1998).

3. R. Nette et al., "Hypotension during hemodialysis results from an impairment of arteriolar tone and left ventricular function," Clin. Nephrol. 63(4), 276-283 (2005).

4. J. Passauer, E. Bussemaker, P. Gross, "Dialysis hypotension: Do we see light at the end of the tunnel? " Nephrol. Dial. Transplant. 13, 3024-3029 (1998).

5. F. M. Vander Sande, J. P. Kooman, K. M. Leunissen, "Intradialytic hypotension-new concepts on an old problem," Nephrol. Dial. Transplant. 15(11), 1746-1748 (2000).

6. G. Ligtenberg, P. J. Blankestijn, H. A. Koomans, "Presyncope during progressive hypovolaemia simulated by lower body negative pressure is not prevented by high-dose naloxone," Nephrol. Dial. Transplant. 13, 398-403 (1998).

7. H. Gesche, "Continuous blood pressure measurement by using the pulse transit time: Comparison to a cuff-based method," Europ. J. Appl. Physiol. 112(1), 309-315 (2012).

8. M. A. Younessi Heravi, M. A. Khalilzadeh, S. Joharinia, "Continous and cuffless blood pressure monitoring using ECG and $\mathrm{SpO} 2$ signals," J. Biomed. Phys. Eng. 4(1), 27-32 (2014). 
9. R. A. Payne, C. N. Symeonides, D. J, Webb, S. R. Maxwell "Pulsetransit time measured from the ECG: An unreliable marker of beat-to-beat blood pressure," J. Appl Physiol. 100: 136-141 (2006)

10. M. Y.- M. Wong, C. C.-Y. Poon, Y.-T. Zhang, "An evaluation of the cuffless blood pressure estimation based on pulse transit time technique: A half year study on normotensive subjects," Cardiovasc. Eng. 9(1), 32-38 (2009).

11. R. Maggi, V. Viscardi, T. Furukawa, M. Brignole, "Non-invasive continuous blood pressure monitoring of tachycardic episodes during interventional electrophysiology," Europace 12(11), 1616-1622 (2010).

12. M. Saban, Two methods for determination of diastolic and systolic pressures in fingers, IEEE 17th Annu. Conf. Engineering in Medicine and Biology Society, IEEE, Montreal, Que (1995).

13. P. Zurek, O. Krejcar, M. Penhaker, M. Cerny, R. Frischer, Continuous Noninvasive Blood Pressure Measurement by Near Infra Red CCD Camera and Pulse Transmit Time Systems, Second Int. Conf. Computer Engineering and Applications (ICCEA), Bali Island, pp. 19-21 March (2010).

14. W. Chen, T. Kobayashi, S. Ichikawa, Y. Takeuchi, T. Togawa, "Continuous estimation of systolic blood pressure using the pulse arrival time and intermittent calibration," Med. Biol Eng. Comput. 38(5), 569-574 (2000).

15. M. Nitzan, B. Khanokh, Y. Slovik, "The difference in pulse transit time to the toe and finger measured by photoplethysmography," Physiol. Meas. 23(1), 85-93 (2002).

16. D. Zheng, A. Murray, "Non-invasive quantification of peripheral arterial volume distensibility and its non-linear relationship with arterial pressure," $J$. Biomech. 42(8), 1032-1037 (2009).

17. M. A. Younessi Heravi, M. A. Khalilzadeh, Designing and Constructing an Optical System to measure Continuous and Cuffless Blood Pressure Using Two Pulse Signals," Iran. J. Med. Phys. 10(4), 204-212 (2014).

18. F. J. Callaghan, C. F. Babbs, J. D. Bourland, L. A. Geddes, "The relationship between arterial pulsewave velocity and pulse frequency at different pressures," J. Med Eng Technol. 8(1), 15-18 (1984).
19. W. Nichols, M. O'Rourke, C. Vlachopoulos, Mcdonald's Blood Flow In Arteries: Theoretical, Experimental and Clinical Principles, CRC Press Boca Raton (2011).

20. P. Shaltis, A. Reisner et al. (2004). A hydrostatic pressure approach to cuffless blood pressure monitoring, Annu. Int. Conf. IEEE Engineering in Medicine and Biology Society, IEMBS'04, IEEE, San Francisco, CA (2004).

21. J. M. Bland, D. G. Altman, "Statistical methods for assessing agreement between two methods of clinical measurement," Lancet 327, 307-310, 1986.

22. D. Franchi, R. Bedini, F. Manfredini, S. Berti, G. Palagi, S. Ghione, A. Ripoli, "Blood pressure evaluation based on arterial pulse wave velocity," Comput. Cardiol. 23, 108-111 (1996).

23. D. B. Newlin, "Relationships of pulse transmission times to pre-ejection period and blood pressure," Psychophysiology 18, 316-321 (1981).

24. M. H. Pollak, P. A. Obrist, "Aortic-radial pulse transit time and ECG qwave to radial pulse wave interval as indices of beat-by-beat blood pressure change," Psychophysiology 20, 21-28 (1983).

25. C. F. Wippermann, D. Schranz, R. G. Huth, "Evaluation of the pulse wave arrival time as a marker for blood pressure changes in critically ill infants and children," J. Clin. Monit. 11, 324-328 (1995).

26. C. Ahlstrom, A. Johansson et al. "Noninvasive investigation of blood pressure changes using the pulse wave transit time: A novel approach in the monitoring of hemodialysis patients," J. Artif. Organs 8(3), 192-197 (2005).

27. D.-K. Jung, G.-R. Kim et al., Changes of pulse wave velocity in arm according to characteristic points of pulse wave, Int. Conf. Convergence Information Technology, Gyongju, South Korea (2007).

28. Y. Kim, J. Lee, Cuffless and non-invasive estimation of a continuous blood pressure based on ptt, 2nd Int. Conf. IEEE Information Technology Convergence and Services (ITCS), Cebu (2010).

29. Zakaria, N. A., N. Sharifmuddin et al., Pulse wave transit time and its relationship with systolic blood pressure, 6th World Congress of Biomechanics (WCB 2010), August 1-6, Springer Singapore, (2010), pp. 15-18. 